American J. of Engineering and Applied Sciences 2 (2): 446-450, 2009

ISSN 1941-7020

(C) 2009 Science Publications

\title{
Iterative Numerical Method of Gate Turn-Off Thyristor: Comparative Study Between $\mathrm{Si}$ and $\mathrm{SiC}$
}

\author{
S.M. Zahim, M. Norainon, J.M. Shawal and R.I.R.M. Taufika \\ Faculty of Electrical and Electronics Engineering, University Malaysia Pahang, \\ Kuantan, Malaysia
}

\begin{abstract}
Problem statement: Silicon (Si) has long been the dominant semiconductor of choice for high-power, high-temperature and high-frequency device applications. However, recently, wide bandgap semiconductors, particularly Silicon Carbide ( $\mathrm{SiC}$ ) has attracted because it offers tremendous benefits over other semiconductor materials in a large number of industrial and military. A change of technology from $\mathrm{Si}$ to $\mathrm{SiC}$ will revolutionize the power electronics and simulation is needed to predict their characteristics. Approach: The system of partial differential equations which forms the Poisson's and continuity equations together with appropriate boundary conditions cannot be solved explicitly in general. Therefore, the solution must be calculated by means of numerical approaches. Application of Finite Element Method (FEM) for semiconductor device simulation is described. It was shown that this method guarantees exact conservation of current both locally and at the device terminals. Finite element forms of Poisson's equation and the electron and hole current continuity equations were derived. Results: This study described the implementation of FEM in order to get the doping profile of the semiconductor devices. The switching simulation, 4H-SiC GTO thyristor was presented and tested numerically by predicting its performance using the 2-D simulator developed in this project. Conclusion: We calculated turn-off time and loss of SiC-GTO and compared with Si-GTO thyristor using 2-dimensional device simulation. Results showed that turn-off time and loss are smaller in SiCGTO thyristor rather than Si-GTO thyristor.
\end{abstract}

Key words: GTO thyristor, finite element method, turn-off time, poisson equation, device simulator, silicon carbide

\section{INTRODUCTION}

The FEM (sometimes referred to as Finite Element Analysis (FEA)) is a numerical technique for finding approximate solutions of partial differential equation as well as of integral equations. The solution approach is based either an approximating system of ordinary differential equations, which are then solved using standard techniques such as Newton Method. It is the objective of this study to describe the application of the method to device simulation. The device which described in this study is Silicon Carbide Gate Turn-Off Thyristor (SiC-GTO Thyristor). The doping profile with the material properties of the device can be modeled. This study specifically focuses on the numerical simulation of the device compare with the common Silicon GTO Thyristor.

The main advantages of the FEM are that conservation laws (e.g., current conservation) are exactly satisfied even by coarse approximations, it is easy to treat irregular geometries, the computational mesh can be graded to be fine in regions to rapid change, local mesh refinement is easier to implement than Finite Difference Method (FDM).

The need for improvement in performance of power electronic systems in many applications has brought about much advancement in $\mathrm{Si}$ technology. Si is the material dominating the electronic industry for high-power, high-temperature and high-frequency device applications. However, recently, wide bandgap semiconductor has superior properties because its offers tremendous benefits over other semiconductor material for power devices. A change of technology from Si to $\mathrm{SiC}$ will revolutionize the power electronics. Hence, the use of $\mathrm{Si}$ devices in high power applications, which require operating in higher temperatures and faster switching speeds, is restricted. In the particular area of power devices, $\mathrm{SiC}$ material has been utilized in order to achieve fast switching time and low switching $\operatorname{loss}^{[1-3]}$.

Corresponding Author: Muhamad Zahim Sujod, Faculty of Electrical and Electronics Engineering,

University Malaysia Pahang, Lebuhraya Tun Razak, 26300 Kuantan, Pahang, Malaysia 
A GTO thyristor is a special type thyristor, a highpower semiconductor device. GTOs as opposed to normal thyristors, are fully controllable switches which can be turned on and off by their third lead, gate lead. Potential uses of GTO includes in the design and production of high-power, high-temperature devices for use in semiconductor electronic switches. The hexagonal lattice structure $4 \mathrm{H}$ and $6 \mathrm{H} \mathrm{SiC}$ are two primary $\mathrm{SiC}$ poly-types widely used in thyristors. Due to its higher electron mobility than that $6 \mathrm{H} \mathrm{SiC}, 4 \mathrm{H} \mathrm{SiC}$ has been chosen as the device material in this study. In addition, $4 \mathrm{H} \mathrm{SiC}$ hole mobility is $30 \%$ higher than $6 \mathrm{H}$ $\mathrm{SiC}$.

SiC-GTO thyristor complimented by the material advantages of $\mathrm{SiC}$ has better characteristics than its $\mathrm{Si}$ counterpart $^{[4]}$. This study presents a comparison study of $4 \mathrm{H} \mathrm{SiC}-\mathrm{GTO}$ thyristor and $\mathrm{Si}-\mathrm{GTO}$ thyristor using numerical simulation. We calculate turn-off time and loss of SiC-GTO thyristor using 2-dimensional device simulation developed along with this research.

\section{MATERIALS AND METHODS}

Semiconductor equation: The semiconductor equations are a set of five equations that govern the behavior of semiconductor materials and devices. The set of equations composed of:

\section{Poisson's equation:}

$\nabla^{2} \psi=\frac{\mathrm{q}}{\varepsilon}\left(\mathrm{n}-\mathrm{p}-\mathrm{N}_{\mathrm{d}}\right)$

\section{Current continuity equations:}

$q \frac{\partial p}{\partial t}=-\tilde{N} \times J_{p}-q R$

$q \frac{\partial n}{\partial t}=\tilde{N} \times J_{n}-q R$

\section{Drift-diffusion equations:}

$$
\begin{aligned}
& J_{p}=q\left(p \tilde{v}_{p}-D_{p} \tilde{N} p\right) \\
& J_{n}=q\left(p \tilde{v}_{n}-D_{n} \tilde{N} n\right)
\end{aligned}
$$

In these equations the three unknown quantities are the space-charge potential ( $\Psi)$, the electron (n) and hole (p) densities, $\mathrm{N}_{\mathrm{d}}$ is the doping densities, the constant $\mathrm{q}$ is the magnitude of electronic charge and $\varepsilon$ is the dielectric permittivity. $\mathrm{J}_{\mathrm{p}}$ and $\mathrm{J}_{\mathrm{n}}$ are the hole and electron current densities. $\mathrm{R}$ is the recombination rate. $\tilde{\mathrm{v}}_{\mathrm{p}}$ and $\tilde{\mathrm{v}}_{\mathrm{n}}$ are the hole and electron drift velocities. $D_{p}$ and $\mathrm{D}_{\mathrm{n}}$ are the hole and electron diffusion coefficients. The diffusion coefficients and drift velocities are electric field dependent and so the equations are nonlinear. The recombination term which is also nonlinear may be approximated by its thermal equilibrium value (Schokley Read Hall Theory) ${ }^{[5]}$.

Finite element equations: To solve (1-5), boundary conditions for the space-charge potential and electron and hole charge carrier densities are required. The finite element equations are derived from (1-3) by multiplying them by $\mathrm{i}(\mathrm{x}, \mathrm{y})$ and integrating over the region $\Omega$ occupied by the device ${ }^{[6]}$.

$$
\begin{aligned}
& \int_{\Omega} \Phi_{\mathrm{i}}(\mathrm{x}, \mathrm{y}) \tilde{\mathrm{N}}^{2} \psi \mathrm{ds}= \\
& -\frac{\mathrm{q}}{\varepsilon} \int_{\Omega}(\mathrm{x}, \mathrm{y})\left(\mathrm{p}-\mathrm{n}-\mathrm{N}_{\mathrm{d}}\right) \mathrm{ds}
\end{aligned}
$$

$$
\begin{aligned}
& \frac{1}{\mathrm{q}} \int_{\Omega} \Phi_{\mathrm{i}}(\mathrm{x}, \mathrm{y}) \tilde{\mathrm{N}} \mathrm{J}_{\mathrm{p}} \mathrm{ds}= \\
& \int_{\Omega} \Phi_{\mathrm{i}}(\mathrm{x}, \mathrm{y})\left(\frac{\partial \mathrm{p}}{\partial \mathrm{t}}+\mathrm{R}\right) \mathrm{ds} \\
& -\frac{1}{\mathrm{q}} \int_{\Omega} \Phi_{\mathrm{i}}(\mathrm{x}, \mathrm{y}) \tilde{\mathrm{N}} \mathrm{J}_{\mathrm{n}} \mathrm{ds}= \\
& \int_{\Omega} \Phi_{\mathrm{i}}(\mathrm{x}, \mathrm{y})\left(\frac{\partial \mathrm{n}}{\partial \mathrm{t}}+\mathrm{R}\right) \mathrm{ds}
\end{aligned}
$$

In computer solution by the finite element method there are four stages:

- Read in (or generate internally) material properties$\mathrm{Si}$ and $\mathrm{SiC}$ ) and element connectivity (mesh)

- Assemble the Eq. 6-8 which the finite element equations and inserting boundary conditions

- Solve the resulting linear Equation

- Repeat iteratively for nonlinear and/or time dependent problems

Mobility models: The Low-Field Mobility Models is used for physical models in the simulation. The Caughey-Thomas approximation is used to model the doping concentration and the temperature. The equations used in the mobility model are given in ${ }^{[6]}$ as: 


$$
\mu_{\mathrm{o}}=\mu_{\min }+\frac{\mu_{\max }-\mu_{\min }}{1+\left(\frac{\mathrm{N}_{\mathrm{D}_{\mathrm{N}}}+\mathrm{N}_{\mathrm{A}}}{\mathrm{N}_{\text {ref }}}\right)^{\alpha}}
$$

Where:

$$
\begin{aligned}
& \mathrm{N}_{\mathrm{D}}+\mathrm{N}_{\mathrm{A}} \quad=\text { The total doping concentration } \\
& \mu_{\max } \text { and } \mu_{\min }=\text { The maximum and minimum motilities } \\
& \text { of electrons and holes } \\
& \mathrm{N}_{\text {ref }} \quad=\text { The doping concentration for } \mathrm{p} \text {-type } \\
& \text { and n-type materials calculated } \\
& \text { empirically } \\
& \alpha \\
& =\text { The curve fitting parameter measure of } \\
& \text { how quickly the mobility changes from } \\
& \mu_{\min } \text { to } \mu_{\max }
\end{aligned}
$$

The fitting parameters are listed in Table 1.

Carrier generation-recombination models: Carrier generation and recombination result from interaction between electrons and other devices, either with the lattice of the material. As the electrons moves from one energy band to another, its gained or lost energy must take some other form and the form of the energy distinguishes various types of generation and recombination Schockley-Read-Hall (SRH) process. In the research, we only consider SRH recombination process among above mentioned carrier generationrecombine processes and it is represented by:

$$
\mathrm{R}=\frac{\mathrm{pn}-\mathrm{n}_{\mathrm{i}}}{\tau_{\mathrm{p}}\left(\mathrm{n}+\mathrm{n}_{\mathrm{i}}\right)+\tau_{\mathrm{n}}\left(\mathrm{p}+\mathrm{n}_{\mathrm{i}}\right)}
$$

Here $\tau_{\mathrm{p}}$ and $\tau_{\mathrm{n}}$ are the hole and electron lifetimes, $\mathrm{n}_{\mathrm{i}}$ is the intrinsic concentration. The values of these used here are shown in Table $1^{[3]}$.

Device design and structure: In order to analyze the switching performances of GTO thyristor, good switching characteristic of the thyristor is the primary goal we want to achieve. At the same time, inside the device such as internal potential distribution, carrier density distribution and current distribution is also properties to be considered.

Figure 1 show a schematic cross section of a halfcell of the structure of the $4 \mathrm{H} \mathrm{SiC-GTO}$ thyristor which we have assumed for our simulation. The GTO thyristor rated is $1200 \mathrm{~V} / 90 \mathrm{~A}$. In the 2-dimensional model, Fig. 2 shows the 2-dimension model of doping profile. Each region of the profile has been calculated using the Gauss distribution, which have 33 segments.
Table 1: Parameters used in mobility models

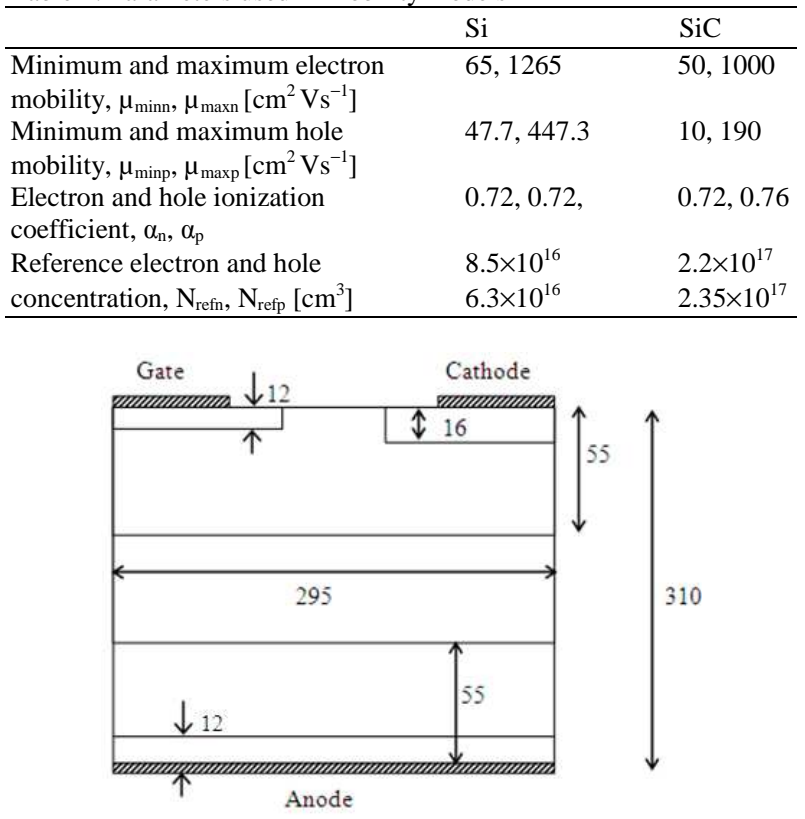

Fig. 1: Schematic of a half-cell of the $4 \mathrm{H} \mathrm{SiC-GTO}$ thyristor (distances in micrometers)

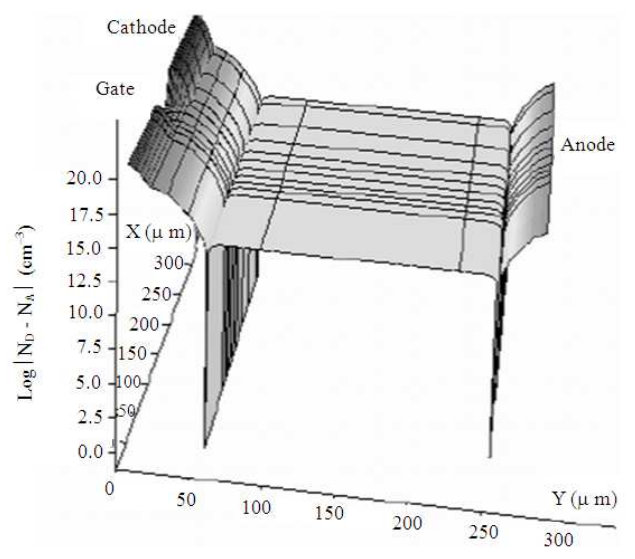

Fig. 2: Doping profile of GTO thyristor

Simulation flow: The simulator described in earlier section and the simulation systems have been implemented by using $\mathrm{C}++$ object-oriented programming language. As mentioned in previous section, this package is used Poisson's equation together with current continuity and drift-diffusion equations to simulate the performances of SiC-GTO thyristor. Figure 3 shows the schematic structure of the simulator. Each phase describes complex process which involves the physical models along with the basic semiconductor equations as the basis to simulate the GTO performances. 
The simulator is controlled by the Material Input Database in each phase. The left line indicates the connection with the material database. Material Input Database is initialized at the initialization process. The basic structure of SiC-GTO thyristor is initialized. The device structure and circuit definitions and additional information like material properties are loaded from the Material Input Database.

In the next step, the device or the circuit and its embedded devices are loaded and analyzed. For each segment of each device the material is determined. In the calculations steps, the basic semiconductor equations along with the physical models are solved by using numerical method, finite element method. The method is a powerful method for solving partial differential equations which involves lots of integral and differential. The method is used because of its approximation to the solution of the equation. In the post processing, the output quantities are calculated from the computed solution.

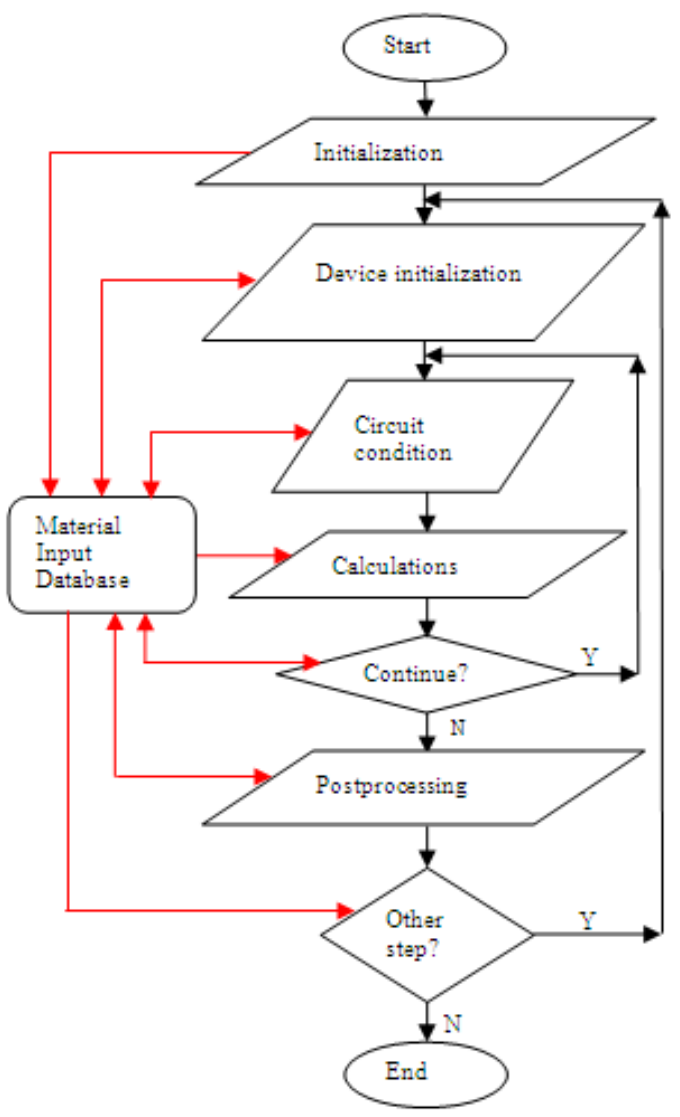

Fig. 3: Schematic structure of the simulator

\section{RESULTS}

Figure 4 and 5 show the switching waveforms of Si-GTO and SiC-GTO, respectively. In the simulation, we raised anode voltage to $100 \mathrm{~V}$, GTOs are turned on (anode current is increased to $10 \mathrm{~A}$ and anode voltage is decreased to $0 \mathrm{~V}$ ). Then they are turned off by negative gate pulse. Anode current decreased to $0 \mathrm{~A}$ and anode voltage recovers to $100 \mathrm{~V}$. We can see large difference at turn-off switching waveforms. We know that turn-off time of SiC-GTO is better than that of Si-GTO. Turn-on time and turn-off time are shown in Table 2 (All units are in $\mu \mathrm{sec}$ ). Result show that switching time of SiCGTO is decreased extremely and the performance of $\mathrm{SiC}$ in GTO is shown in the storage time, fall time and tail time.

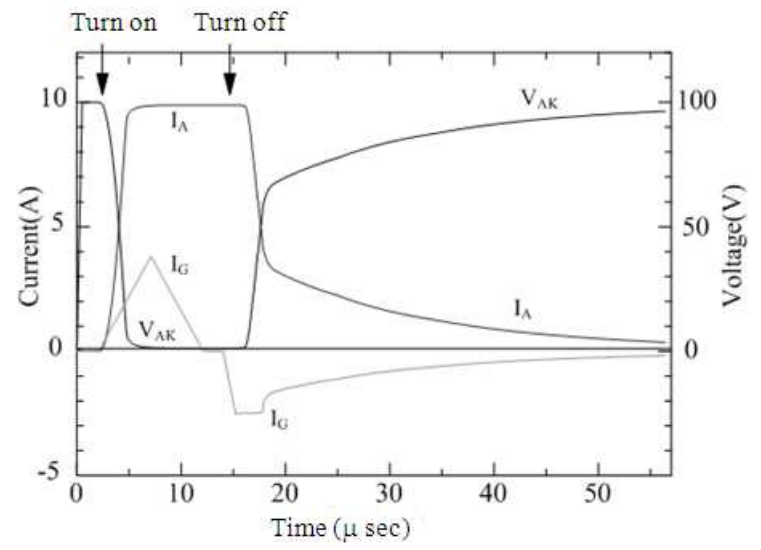

Fig. 4: Switching waveform of Si-GTO thyristor

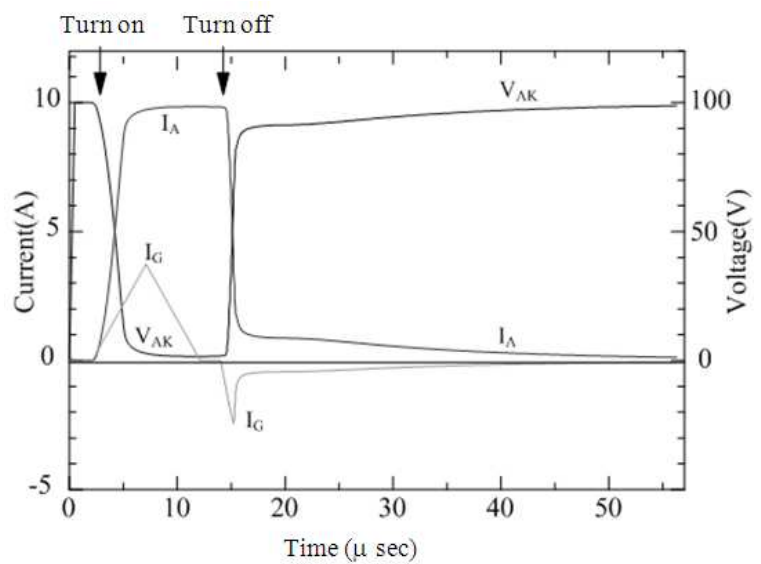

Fig. 5: Switching waveform of SiC-GTO thyristor 
Table 2: Switching time of Si-GTO thyristor and SiC-GTO thyristor

\begin{tabular}{lrr}
\hline & Si-GTO & SiC-GTO \\
\hline Turn-on time & 3.00 & 3.30 \\
Delay time & 1.45 & 1.45 \\
Rise time & 1.55 & 1.85 \\
Turn-off time & 36.20 & 17.80 \\
Storage time & 2.60 & 0.70 \\
Fall time & 10.00 & 0.70 \\
Tail time & 23.60 & 16.40 \\
Switching time & 39.20 & 21.10 \\
\hline
\end{tabular}

\section{CONCLUSION}

In this study, the numerical method of solving semiconductor equations were introduced, which is finite element method. The finite element method can be applied successfully to semiconductor device simulation despite the nonlinear of the governing equations. A detailed derivation has been given by applying divergence theorem into the semiconductor equations. The simulation tool would be the new technology in semiconductor device and simulation in semiconductor industries. By using the simulator, we compared the switching waveforms of usual Si-GTO thyristor and new SiC-GTO thyristor. Turn-off time is smaller in the case of SiC-GTO thyristor than in that SiGTO thyristor.

\section{ACKNOWLEDGEMENT}

The researchers thank the Ministry of Higher Education Malaysia that funded the project.

\section{REFERENCES}

1. Ruff, M., H. Mitlehner and R. Helbig, 1994. SiC devices, physics and numerical simulation. IEEE Trans. Elect. Device, 41: 1041-1054. DOI: $10.1109 / 16.293319$
2. Sehadri, S., J.B. Casady, A.K. Agrawal, R.R. Siergiej, L.B. Rowland, P.A. Sanger, C.D Brandt, J. Barrow, D. Piccone, R. Rodrigues and T. Hansen, 1998. Turn-off characteristic of $1000 \mathrm{~V}$ SiC GTO thyristor. Proceeding of 1998 International Symposium on Power Devices and ICs, June 3-6, IEEE Xplore Press, Kyoto, Japan, pp: 131-134. DOI: 10.1109/ISPSD.1998.702653

3 Huang, A.Q. and B. Zhang, 2000. Comparing SiC switching power devices, MOSFET, NPN transistor and GTO thyristor. Solid State Elect., 44: $325-340 . \quad$ DOI: $10.1016 / \mathrm{S} 0038-$ 1101(99)00239-7

4 Agrawal, A.K., J.B. Casady, L.B. Rowland, S. Seshadi, R.R. Sieraj, W.F. Valek and C.D. Brandt, 1997. $700 \mathrm{kV}$ Asymmetrical 4H-SiC Gate Turn-Off Thyristors. IEEE Elect. Devices Lett., 18: 518-520. DOI: $10.1109 / 55.641431$

5. Scharfetter, D.L. and H.K. Gummel, 1969. Largesignal analysis of a silicon. IEEE Trans. Elect. Devices, $\quad 16: \quad 64-77$. http://ieeexplore.ieee.org/xpl/freeabs_all.jsp?arnum ber $=1475609$

6. Sakata, H. and M.Z. Sujod, 2002. Device simulation of SiC-GTO. Proceedings of Power Conversion Conference, Apr. 2-5, IEEE Xplore Press, Osaka, Japan, pp: 220-225. DOI: 10.1109/PCC.2002.998551 